\title{
Is there an outcome penalty linked to guideline-based indications for valvular surgery? Early and long-term analysis of patients with organic mitral regurgitation
}

\author{
Maurice Enriquez-Sarano, MD, ${ }^{\mathrm{a}}$ Rakesh M. Suri, MD, DPhil, ${ }^{\mathrm{b}}$ Marie-Annick Clavel, DVM, PhD, ${ }^{\mathrm{a}}$ \\ Francesca Mantovani, MD, ${ }^{\mathrm{a}}$ Hector I. Michelena, MD, ${ }^{\mathrm{a}}$ Sorin Pislaru, MD, ${ }^{\mathrm{a}}$ Douglas W. Mahoney, MS, ${ }^{\mathrm{c}}$ and \\ Hartzell V. Schaff, MD
}

\begin{abstract}
Objective: The timing of surgical correction of mitral regurgitation remains controversial. A major source of dispute regards the potential short- and longterm postoperative outcome penalty associated with the type of guideline-based indication for surgery.

Methods: Between 1990 and 2000, 1512 patients (aged $64 \pm 14$ years, mitral prolapse in $89 \%$, valve repair in $88 \%$ ) underwent surgical correction of pure organic mitral regurgitation. Patients were stratified according to surgical indication into class I triggers (ClassI-T: heart failure symptoms, ejection fraction $<60 \%$, end-systolic diameter $\geq 40 \mathrm{~mm}, \mathrm{n}=794$ ), class II triggers based on clinical complications (ClassII-CompT: atrial fibrillation or pulmonary hypertension, $\mathrm{n}=195$ ), or early class II triggers based on a combination of severe mitral regurgitation and high probability of valve repair (ClassII-EarlyT: $n=523$ ).
\end{abstract}

Results: Operative mortality was highest with ClassI-T $(1.1 \%$ vs $0 \%$ and $0 \%$, $P=.016)$. Long-term survival was lower with ClassI-T $(15$-year $42 \% \pm 2 \%$; adjusted hazard ratio [HR], 1.89; 95\% confidence interval [CI], 1.53-2.34; $P<.0001$ ) and ClassII-CompT (15-year 53\% $\pm 4 \%$, adjusted HR, 1.39; 95\% CI, 1.04-1.84; $P=.027$ ) versus ClassII-EarlyT (15-year 70\% $\pm 3 \%$, $P<.0001)$. Postoperative excess mortality with ClassI-T and ClassII-CompT was confirmed by age stratification, inverse probability weighting, and expected survival adjustment. Excess postoperative heart failure was high with ClassI-T (adjusted HR, 2.49; 95\% CI, 1.82-3.47; $P<.0001$ ) and ClassII-CompT (adjusted HR, $1.98 ; 95 \%$ CI, $1.30-3.00 ; P=.002)$.

Conclusions: The type of guideline-based indication for surgical correction of organic mitral regurgitation is associated with profound outcome consequences on long-term postoperative mortality and heart failure, despite low operative risk and high repair rates. Conversely, surgical correction of severe mitral regurgitation based on high probability of repair (ClassII-EarlyT) is associated with improved survival and low heart failure risk and should be the preferred strategy in valve centers offering low operative risk and high repair rates. (J Thorac Cardiovasc Surg 2015;150:50-8)

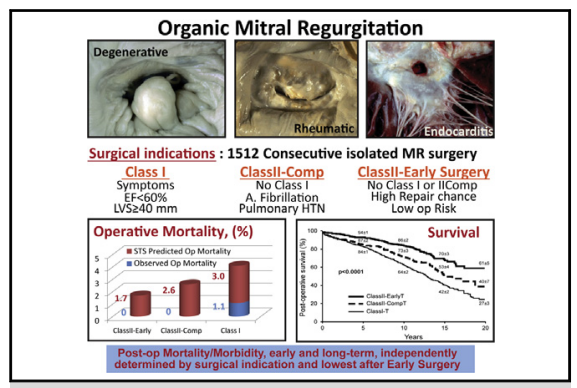

From top to bottom: lesions of organic MR, classes of indications, and outcomes by indication.

\section{Central Message}

Guideline triggers for MR surgery based on symptoms and complications are linked to excess postoperative mortality/morbidity versus early surgery. Early repair should be preferred to rescue surgery in patients with MR.

\section{Perspective}

Controversy regarding the timing of surgery for organic MR hinges on the assumption that guideline-based triggers allow safe outcomes. Surgical improvements, higher repair rates, and lower operative mortality may support this assumption. In 1512 patients undergoing operation for organic MR (repair $88 \%$, operative mortality $0.6 \%$ ), triggers for class I (symptoms, $\mathrm{EF}<60 \%$, end-systolic diameter $\geq 40 \mathrm{~mm}$ ) were independently associated with doubling of long-term death/heart failure risk, and triggers for class II (atrial fibrillation, pulmonary hypertension) were associated with a $40 \%$ increase in risk. Thus, these triggers lead to rescue surgery when present initially, but this is not the preferred surgical timing. Early surgery, performed in centers with high repair rates and low risk, with outstanding outcomes should be the preferred strategy.

See Editorial page 4.
From the Divisions of ${ }^{\mathrm{a} C}$ ardiovascular Diseases and Internal Medicine, ${ }^{\mathrm{b}} \mathrm{Cardiac}$ Surgery, and ${ }^{\mathrm{c} H e a l t h}$ Science Research, Mayo Clinic, Rochester, Minn. The study was funded by the Mayo Clinic Foundation.

Received for publication Jan 26, 2015; revisions received March 24, 2015; accepted for publication April 3, 2015; available ahead of print May 16, 2015

\footnotetext{
Address for reprints: Maurice Enriquez-Sarano, MD, Division of Cardiovascular Diseases and Internal Medicine, Mayo Clinic, 200 First St SW, Rochester, MN 55905 (E-mail: sarano.maurice@mayo.edu).

$0022-5223 / \$ 36.00$

Copyright (c) 2015 by The American Association for Thoracic Surgery

http://dx.doi.org/10.1016/j.jtcvs.2015.04.009
} 


$$
\begin{aligned}
& \text { Abbreviations and Acronyms } \\
& \begin{aligned}
\text { CABG } & =\text { coronary artery bypass grafting } \\
\text { CHF } & =\text { congestive heart failure } \\
\text { CI } & =\text { confidence interval } \\
\text { EF } & =\text { ejection fraction } \\
\text { HR } & =\text { hazard ratio } \\
\text { IPW } & =\text { inverse probability weight } \\
\text { LV } & =\text { left ventricular } \\
\text { MR } & =\text { mitral regurgitation } \\
\text { STS } & =\text { Society of Thoracic Surgeons }
\end{aligned}
\end{aligned}
$$

Guidelines for the clinical management of organic mitral regurgitation (MR) are similar between $\mathrm{US}^{1}$ and European ${ }^{2}$ versions. These guidelines define the triggers for surgical indications; class I triggers (heart failure symptoms, low left ventricular $[\mathrm{LV}]$ ejection fraction $[\mathrm{EF}]$, or large end-systolic dimension) generally mandate prompt operation, whereas class II triggers, whether guided by clinical complications (atrial fibrillation, pulmonary hypertension) or high MR severity and repairability (early surgery), are less rigid with leeway for clinical judgment. ${ }^{1,2}$ Although the guidelines vary little between versions, they are based on level $\mathrm{C}$ evidence, that is, mainly expert opinions. Thus, an intense debate persists regarding the best timing for MR surgery.,

Class I triggers for MR surgery are based on studies of the 1990s analyzing outcomes of patients who underwent surgery in the $1980 \mathrm{~s}^{5-7}$ Symptoms, ${ }^{6,8}$ decreased $\mathrm{EF}^{5,9}$ or large LV end-systolic diameter ${ }^{10}$ were all mentioned as affecting postoperative outcome, suggesting a penalty related to these triggers. Likewise, class II triggers considered as "minor" complications may have long-term consequences. ${ }^{11,12}$ However, improvements have occurred since these pilot studies; there has been a decline in operative mortality and a marked increase in performance of valve repair, ${ }^{13}$ which may protect against the postoperative consequences of these triggers. ${ }^{14}$ Thus, whether long-term surgical outcome, in the era of valve repair and low operative risk, is similar irrespective of surgical triggers or is affected by triggers indicating MR surgery is unclear and may be crucial to formulate clinical guidelines. Thus, it is essential to analyze long-term outcome according to the triggers that led to surgery. To address this conundrum, new data are warranted, and we analyzed consecutive patients undergoing operation for organic MR between 1990 and 2000 to obtain a sufficiently long follow-up. We examined the null hypothesis that indications for organic MR surgery were not independently associated with differences in clinical outcome, particularly long-term survival.

\section{MATERIALS AND METHODS \\ Subjects}

Eligible patients were those who underwent mitral surgery at the Mayo Clinic (Rochester, Minn) for pure isolated organic (surgically verified intrinsic structural mitral disease) MR between January 1, 1990, and December 31, 2000. We excluded patients associated mitral stenosis, concomitant aortic valve replacement/repair or tricuspid valve replacement, previous valve replacement/repair or congenital heart disease surgery, or with pericardial, myocardial intrinsic disease or ischemic MR with or without papillary muscle rupture. The study was approved by the Mayo Clinic Institutional Review board as a low-risk study.

\section{Baseline Characteristics}

Clinical symptoms of recent murmur ( $<6$ months), comorbidity, blood pressure, and heart rate were noted as classic MR signs. Electrocardiogram allowed the diagnosis of atrial fibrillation. Doppler echocardiography measured LV diameters and EF, quantified MR and pulmonary pressure, and determined MR cause, mechanisms, and associated cardiac conditions. ${ }^{15}$ Catheterization diagnosed obstructive coronary disease ( $>50 \%$ left main, $>70 \%$ other segments). With the entire information available, a risk score was calculated using the Society of Thoracic Surgeons (STS) algorithm based on the coefficient provided by the Society in 2013. ${ }^{16}$ Indication for surgery relied on shared decision-making by the patient, cardiologist, and cardiac surgeon. We classified surgical indications by guideline-based preoperative characteristics as class I (heart failure symptoms, $\mathrm{EF}<60 \%$, or end-systolic $\mathrm{LV}$ diameter $\geq 40 \mathrm{~mm}$; ClassI-T group) and as class II due to clinical "minor" complication (no ClassI-T but with atrial fibrillation or pulmonary hypertension systolic pulmonary pressure $\geq 50 \mathrm{~mm} \mathrm{Hg}$; ClassII-CompT group). Patients with severe MR, no other surgical trigger, and undergoing early surgery with a high probability of valve repair comprised the ClassII-EarlyT group.

\section{Operative Characteristics}

Operative reports verified anatomic mitral lesions. Surgery performed (repair vs replacement), concomitant coronary artery bypass grafting ( $\mathrm{CABG}$ ), and duration of cardiopulmonary bypass were recorded. Residual MR after resumption of normal circulation (by transesophageal echocardiography or double-sampling dye curves) was systematically noted. Postoperative complications recorded were perioperative mortality (within 30 days of surgery or same hospitalization), need for circulatory support beyond the first postoperative day, intra-aortic balloon pump, low cardiac output syndrome postoperatively, and renal failure postoperatively. Finally, the total number of days spent in the hospital was counted.

\section{Long-Term Clinical Outcome}

Follow-up after discharge was obtained through clinical visits at our institution or affiliated clinics and hospitals and through questionnaires to patients. Data were collected throughout the follow-up period to detect transient events. Additional information was obtained using communication with personal physicians, patients, and next of kin, or use of death or autopsy certificates. Thus, we obtained follow-up until death or at least 10 years postoperatively in $92 \%$ of patients. The main end point was long-term postoperative survival as a simple categoric measure of outcome. Secondary end points were postoperative occurrence of heart failure defined using Framingham signs and symptoms criteria ${ }^{17}$ as cardiac-specific measures of outcome and occurrence of the combined end point of death or heart failure.

\section{Statistical Analysis}

Baseline characteristics are described as mean \pm standard deviation for continuous variables and percentages for categoric variables. Comparisons between groups were made using analysis of variance with Tukey post hoc testing for continuous variables and chi-square or Fisher exact test as necessary for categoric variables. Long-term survival and event rates were summarized using the Kaplan-Meier method for estimating 
rates \pm standard errors and compared using the log-rank test. Cox proportional hazard models assessed hazard ratios (HRs) and $95 \%$ confidence intervals (CIs) attached to surgical indication groups by using 2 dummy variables for the ClassI-T and ClassII-CompT groups (therefore compared with the reference ClassII-EarlyT group). We used univariable models with these dummy variables and multivariable models adjusting for age, sex, diabetes, creatinine, obstructive coronary disease, valve repair, CABG, and STS score, all predictive of survival in mitral diseases. The same adjustment variables were used in logistic models analyzing early postoperative complications. For long-term survival analysis, we also calculated inverse probability weights (IPWs) from predicted probabilities of trigger class assignment (ClassI-T, ClassII-CompT, and ClassII-EarlyT) using age, sex, diabetes, obstructive coronary disease, creatinine, valve repair, $\mathrm{CABG}$, and STS score. The IPW weight was then used within the Cox proportional hazards regression model to generate a weighted population with a balanced distribution of covariates for unbiased estimates of death hazard for ClassI-T and ClassII-CompT compared with ClassII-EarlyT. The expected survivals relative to age- and sex-expected survivals (US whites) were used to estimate HRs for group variables adjusting for each patient's expected survival. Variables used to construct indication groups were not adjusted to avoid tautological inferences. Statistical analysis was performed with JMP 10 and SAS 9 software (SAS Institute Inc, Chicago, Ill).

\section{RESULTS}

Among 1512 patients fulfilling the eligibility criteria, most $(\mathrm{n}=794,52.5 \%)$ had 1 or more class I trigger (heart failure symptoms, $\mathrm{EF}<60 \%$, end-systolic diameter $\geq 40 \mathrm{~mm}$ ) and formed the ClassI-T group, whereas isolated ClassII-CompT (atrial fibrillation, pulmonary hypertension) was a smaller group $(\mathrm{n}=195,13 \%)$ and the ClassII-EarlyT group included 523 patients $(34.5 \%)$. Overall, MR cause was rheumatic in $5 \%$ of patients, endocarditic in $5 \%$ of patients, degenerative (myxomatous) in $86 \%$ of patients, and miscellaneous organic (primary) in $3 \%$ of patients, and the MR mechanism was a valvular prolapse in $89 \%$ of patients.

\section{Baseline Characteristics}

The baseline characteristics of the entire population are summarized in the left column of Table 1 and are typical of patients undergoing MR surgery at our center and nationally ${ }^{18}$ (aged 64 years, and the majority are men). Comparison of the 3 groups of surgical indications (Table 1) shows expected differences due to triggers used. Also, patients in the ClassI-T and ClassII-CompT groups were older and less often had recent murmur. In the ClassI-T group, there was also a higher prevalence of history of myocardial infarction, coronary disease, hypertension, and diabetes. In view of these differences, an integrator of risk such as the STS predicted mortality score showed modest but significant differences between groups. After inverse probability weighting, no significant difference in baseline characteristics persisted (Table 1, right column).

\section{Operative Characteristics}

The overall operative procedures are listed in the left column of Table 2, revealing relatively short cardiopulmonary bypass times. CABG was performed in approximately one quarter of patients, and valve repair was performed in approximately $90 \%$ of patients. The ClassII-EarlyT group had the highest repair rate and lowest $\mathrm{CABG}$ requirement with shortest time on cardiopulmonary bypass.

For immediate postoperative outcomes, the observed operative mortality of $0.6 \%$ was lower than $2.5 \%$ predicted. ClassI-T was the only group with operative mortality, lower than predicted but higher than in the other groups. Postprocedural MR grade was similar irrespective of surgical indication. Outcome measures (low cardiac output, requirement for inotropic treatment, intra-aortic balloon pump, and total days in-hospital) were worse in the ClassI-T group. After adjusting for age, sex, creatinine, diabetes, coronary disease, CABG, valve repair, and STS score, operative mortality $(P<.01)$, low cardiac output $(P<.001)$, and requirement for inotropes $(P<.001)$ remained higher in the ClassI-T group than in the ClassII-EarlyT group, whereas hospital stay was higher in the ClassI-T group $(P<.0001)$ and ClassII-CompT group $(P<.01)$ than in the ClassII-EarlyT group.

\section{Postoperative Survival}

During a follow-up of $10.8 \pm 4.6$ years, 617 deaths were recorded. Survival in the entire cohort at $5,10,15$, and 20 years was $88 \% \pm 1 \%, 73 \% \pm 1 \%, 52 \% \pm 2 \%$, and $37 \% \pm 3 \%$, respectively, with 22 patients remaining at risk at 20 years. Survival was considerably different according to indications (Figure 1), with 15-year survival of $42 \% \pm 2 \%$ in the ClassI-T group, $53 \% \pm 4 \%$ in the ClassII-CompT group, and $70 \% \pm 3 \%$ in the ClassII-EarlyT group $(P<.0001)$. Unadjusted HRs of mortality linked to indications (Table 3 ) were 2.56 for the ClassI-T group and 1.77 for the ClassII-CompT group versus ClassII-EarlyT (both $P<.0001$ ).

To compare survival between trigger groups, particularly in terms of age, several methods were used. First, in Cox proportional hazards multivariable analysis, mortality risk attached to ClassI-T and ClassII-CompT remained highly significant (Table 3, right), indicating an approximate doubling of mortality with ClassI-T $(P<.0001)$ and approximately $40 \%$ increased mortality with ClassII-CompT versus ClassII-EarlyT. Second, stratifying by age ( $<65$ and $\geq 65$ years) allowed comparison of similarly aged patients between ClassI-T and ClassIIEarlyT groups (stratified ClassII-CompT subgroups were too small). For patients aged less than 65 years, these 2 groups had similar age $(52 \pm 10$ years vs $51 \pm 10$ years, $P=.27$ ), but 15 -year survival was lower in the ClassI-T group than in the ClassII-EarlyT group $(70 \% \pm 3 \%$ vs $84 \% \pm 3 \%, P<.001)$. Likewise, for patients aged 65 years or more, the groups had comparable age (74.3 \pm 6 years vs $73.3 \pm 5$ years, $P=.06)$, but 15 -year survival was lower in the ClassI-T group than in 
TABLE 1. Baseline characteristics

\begin{tabular}{|c|c|c|c|c|c|c|}
\hline & $\begin{array}{c}\text { Overall series } \\
(\mathbf{n}=\mathbf{1 5 1 2})\end{array}$ & $\begin{array}{c}\text { ClassI-T } \\
(n=794)\end{array}$ & $\begin{array}{l}\text { ClassII-CompT } \\
\quad(\mathbf{n}=195)\end{array}$ & $\begin{array}{c}\text { ClassII-EarlyT } \\
\quad(\mathbf{n}=\mathbf{5 2 3})\end{array}$ & $P$ value & $\begin{array}{c}\text { Post-IPW } \\
P \text { value }\end{array}$ \\
\hline \multicolumn{7}{|l|}{ Trigger-related characteristics } \\
\hline Heart failure symptoms, \% & 35 & 63 & 0 & 0 & .0001 & - \\
\hline Atrial fibrillation, $\%$ & 27 & 37 & 63 & 0 & .0001 & - \\
\hline Recent murmur, \% & 34 & 30 & 24 & 44 & .0001 & - \\
\hline $\mathrm{EF}, \%$ & $62 \pm 10$ & $58 \pm 10$ & $68 \pm 5$ & $68 \pm 5$ & .0001 & - \\
\hline LVS, mm & $36 \pm 7$ & $39 \pm 7$ & $32 \pm 4$ & $33 \pm 4$ & .0001 & - \\
\hline LVD, mm & $59 \pm 8$ & $60 \pm 8$ & $57 \pm 7$ & $58 \pm 6$ & .0001 & - \\
\hline \multicolumn{7}{|l|}{ Nontrigger baseline characteristics } \\
\hline Age, y & $64 \pm 14$ & $66 \pm 13$ & $66 \pm 11$ & $60 \pm 14$ & .0001 & \\
\hline Mean age after IPW, y & 66 & 64 & 64 & 68 & & .19 \\
\hline Male sex, \% & 66 & 61 & 67 & 74 & .0001 & \\
\hline Male sex after IPW, \% & 68 & 68 & 67 & 70 & & .87 \\
\hline Smoking history, $\%$ & 45 & 47 & 48 & 42 & .19 & .52 \\
\hline History of MI, \% & 6 & 8 & 4 & 4 & .005 & .18 \\
\hline Obstructive CAD, \% & 28 & 30 & 30 & 24 & .04 & .17 \\
\hline History of HTN, \% & 36 & 39 & 45 & 27 & .0001 & .25 \\
\hline Systolic BP, mm Hg & $131 \pm 19$ & $129 \pm 20$ & $132 \pm 19$ & $131 \pm 19$ & .05 & .12 \\
\hline Cholesterol, mg/dL & $200 \pm 40$ & $197 \pm 42$ & $200 \pm 37$ & $202 \pm 38$ & .14 & .78 \\
\hline Creatinine, mg/dL & $1.21 \pm 0.51$ & $1.26 \pm 0.67$ & $1.17 \pm 0.26$ & $1.13 \pm 0.20$ & .0001 & .07 \\
\hline Weight, $\mathrm{kg}$ & $77 \pm 16$ & $77 \pm 16$ & $76 \pm 18$ & $77 \pm 15$ & .78 & .88 \\
\hline Diabetes, $\%$ & 5.8 & 7.7 & 4.6 & 3.4 & .003 & .17 \\
\hline STS score for operative mortality & $2.5 \pm 2.4$ & $3.0 \pm 2.9$ & $2.6 \pm 1.7$ & $1.7 \pm 1.5$ & .0001 & .14 \\
\hline $\mathrm{ERO}, \mathrm{mm}^{2}$ & $57 \pm 29$ & $58 \pm 32$ & $56 \pm 28$ & $55 \pm 25$ & .40 & - \\
\hline Regurgitant volume, $\mathrm{mL} / \mathrm{beat}$ & $92 \pm 44$ & $90 \pm 44$ & $92 \pm 49$ & $96 \pm 41$ & .31 & - \\
\hline MVP, \% & 89 & 86 & 91 & 95 & .0001 & - \\
\hline
\end{tabular}

$I P W$, Inverse probability weight; $E F$, ejection fraction; $L V S$, left ventricular end-systolic diameter; $L V D$, left ventricular end-diastolic diameter; $M I$, myocardial infarction; $C A D$, coronary artery disease; $H T N$, hypertension; $B P$, blood pressure; $S T S$, Society for Thoracic Surgeons; $E R O$, effective regurgitant orifice; $M V P$, mitral valve prolapse.

the ClassII-EarlyT group $(26 \% \pm 2 \%$ vs $50 \% \pm 5 \%$, $P<.0001)$. Third, IPW was used to comprehensively balance the baseline characteristics between groups with weighing (Table 1) and to compare outcomes. IPW showed that versus ClassII-EarlyT, ClassII-CompT was associated with excess mortality (HR, 1.36; 95\% CI, 1.005-1.85; $P=.046$ ), and ClassI-T had an even higher excess risk (HR, 1.78; 95\% CI, 1.43-2.21; $P<.0001$ ), similar to standard Cox proportional hazard analysis (Table 3). Fourth, by using expected mortality (age and sex matched), excess mortality linked to ClassII-CompT (HR, 1.33; 95\% CI, 1.002-1.77; $P=.048)$ and ClassI-T
(HR, 1.93; 95\% CI, 1.57-2.36; $P<.0001)$ versus ClassII-EarlyT remained similar. Finally, analysis restricted to valve repair showed 10-year postoperative survival of $88 \% \pm 2 \%$ in ClassII-EarlyT versus $75 \% \pm 3 \%$ in ClassII-CompT and $67 \% \pm 2 \%$ in ClassI-T $(P<.0001)$. Compared with ClassII-EarlyT adjusted HR for ClassI-T, 1.86; 95\% CI, 1.48-2.37; $P<.000$, and adjusted HR for ClassII-CompT, 1.37; $95 \%$ CI, $0.995-1.87 ; P=.054$, were almost identical to those of the entire cohort. Thus, all methods used to equitably compare the postoperative survival between trigger groups show that ClassII-CompT incurred $30 \%$ to $40 \%$

TABLE 2. Operative procedures and perioperative outcomes

\begin{tabular}{lccccc}
\hline & Overall series $(\mathbf{n}=\mathbf{1 5 1 2})$ & ClassI-T $(\mathbf{n}=\mathbf{7 9 4})$ & ClassII-CompT $(\mathbf{n}=\mathbf{1 9 5})$ & ClassII-EarlyT $(\mathbf{n}=\mathbf{5 2 3})$ & $\boldsymbol{P}$ value \\
\hline Bypass time, min & $78 \pm 54$ & $83 \pm 53$ & $82 \pm 41$ & $68 \pm 57$ \\
CABG, \% & 26 & 28 & 29 & .0001 \\
Valve repair, \% & 88 & 86 & 88 & .01 \\
Operative mortality, \% & 0.6 & 1.1 & 0 & 91 & .02 \\
Low cardiac output, \% & 3 & 4 & 1.5 & 0 & .016 \\
MR grade postoperatively & $0.17 \pm 0.4$ & $0.17 \pm 0.4$ & $0.20 \pm 0.4$ & .02 \\
IABP required, \% & 1.7 & 2.4 & 1.5 & $0.14 \pm 0.4$ \\
Prolonged inotropes, $\%$ & 13 & 18 & $10.5 \pm 8.3$ & .19 \\
Total d in hospital & $9.8 \pm 6.5$ & $10.6 \pm 7.1$ & .07 & .00 \\
\hline
\end{tabular}

$C A B G$, Coronary artery bypass grafting; $M R$, mitral regurgitation; $I A B P$, intra-aortic balloon pump. 


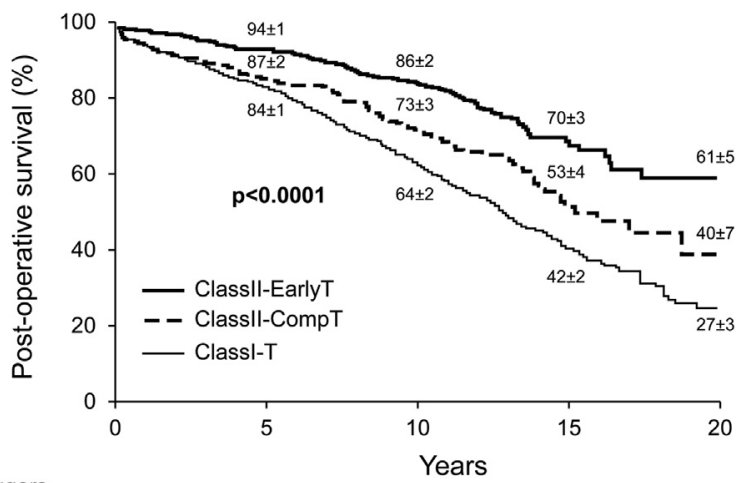

Triggers

Classll-EarlyT 523 ClassII-CompT 195 Classl-T 794
403

127

461
68
38

135

(Table 3) were 3.28 for ClassI-T and 2.45 for ClassII-CompT versus ClassII-EarlyT (both $P<.0001$ ). After multivariate adjustment, the risk attached to triggers remained highly significant (Table 2 , right), approximately 2.5 increased risk in ClassI-T $(P<.0001)$ and approximately double the risk in ClassII-CompT versus ClassII-EarlyT.

During follow-up, 726 patients reached the combined end point of mortality or heart failure with an incidence at 5,10 , 15 , and 20 years of $18 \% \pm 1 \%, 36 \% \pm 1 \%, 55 \% \pm 2 \%$, and $72 \% \pm 3 \%$, respectively. The death/CHF rate was considerably different according to indication and class (Figure 2, right) at 15 years: $65 \% \pm 2 \%$ in ClassI-T, $55 \% \pm 4 \%$ in ClassII-CompT, and $37 \% \pm 3 \%$ in ClassII-EarlyT $(P<.0001)$. Unadjusted and adjusted risk ratios of death/CHF (Table 3) show considerable risk in ClassI-T (adjusted HR, 1.90; 95\% CI, 1.58-2.31; $P<.0001$ ) and notable risk with ClassII-CompT (adjusted HR, 1.53; 95\% CI, 1.18-1.98; $P<.001)$ compared with ClassII-EarlyT.

Analysis restricted to patients undergoing valve repair showed postoperative 10-year death/CHF of $16 \% \pm 2 \%$ in ClassII-EarlyT versus $37 \% \pm 4 \%$ in ClassII-CompT and $43 \% \pm 2 \%$ in ClassI-T $(P<.0001)$ with adjusted risk ratios in ClassI-T $(1.95 ; 95 \%$ CI, 1.57-2.42; $P<.0001)$ and ClassII-CompT (1.58; 95\% CI, 1.19-2.09; $P<.01)$ similar to the overall series. Adjusted HRs stratified by age remained high in ClassI-T in patients aged less than 65 years $(2.19 ; 95 \% \mathrm{CI}, 1.35-3.63 ; P=.001)$ and 65 years or more $(1.78 ; 95 \% \mathrm{CI}, 1.45-2.20 ; P<.0001)$ compared with the ClassII-EarlyT group. For ClassII-CompT, excess risk was also notable in younger $(2.07 ; 95 \% \mathrm{CI}$, $0.98-4.14 ; P=.055)$ and older $(1.35 ; 95 \% \mathrm{CI}, 1.02-1.77$; $P=.037)$ subsets.

\section{DISCUSSION}

The present series is the first to analyze outcomes after surgical treatment of MR according to guideline-based $27 \% \pm 4 \%$ in ClassII-CompT, and $15 \% \pm 3 \%$ in

TABLE 3. Association between clinical triggers for surgery and clinical outcome

\begin{tabular}{|c|c|c|c|c|c|c|}
\hline \multirow[b]{2}{*}{ End points } & \multicolumn{3}{|c|}{ Univariable analysis } & \multicolumn{3}{|c|}{ Multivariable analysis* } \\
\hline & $\mathbf{R R}$ & 95\% CI & $P$ value & $\mathbf{R R}$ & 95\% CI & $P$ value \\
\hline \multicolumn{7}{|c|}{ Survival after surgery } \\
\hline ClassI-T & 2.56 & $2.10-3.15$ & .0001 & 1.89 & $1.53-2.34$ & .0001 \\
\hline ClassII-CompT & 1.77 & $1.32-2.35$ & .0001 & 1.39 & $1.04-1.84$ & .027 \\
\hline \multicolumn{7}{|l|}{$\mathrm{CHF}$ after surgery } \\
\hline ClassI-T & 3.28 & $2.42-4.52$ & .0001 & 2.49 & $1.82-3.47$ & .0001 \\
\hline ClassII-CompT & 2.45 & $1.61-3.70$ & .0001 & 1.98 & $1.30-3.00$ & .002 \\
\hline \multicolumn{7}{|l|}{ Death or CHF } \\
\hline ClassI-T & 2.57 & $2.14-3.10$ & .0001 & 1.90 & $1.58-2.31$ & .0001 \\
\hline ClassII-CompT & 1.96 & $1.52-2.53$ & .0001 & 1.53 & $1.18-1.98$ & .001 \\
\hline
\end{tabular}

$R R$, Risk ratio; $C I$, confidence interval; $C H F$, congestive heart failure. *Adjusted for age at surgery, sex, diabetes, presence of obstructive coronary disease, creatinine, valve repair performance, CABG performance, and STS score with reference using the early surgery (ClassII-EarlyT) group. ClassI-T is the group of patients with class I triggers, and ClassII-CompT is the group of patients with isolated atrial fibrillation or pulmonary hypertension. 

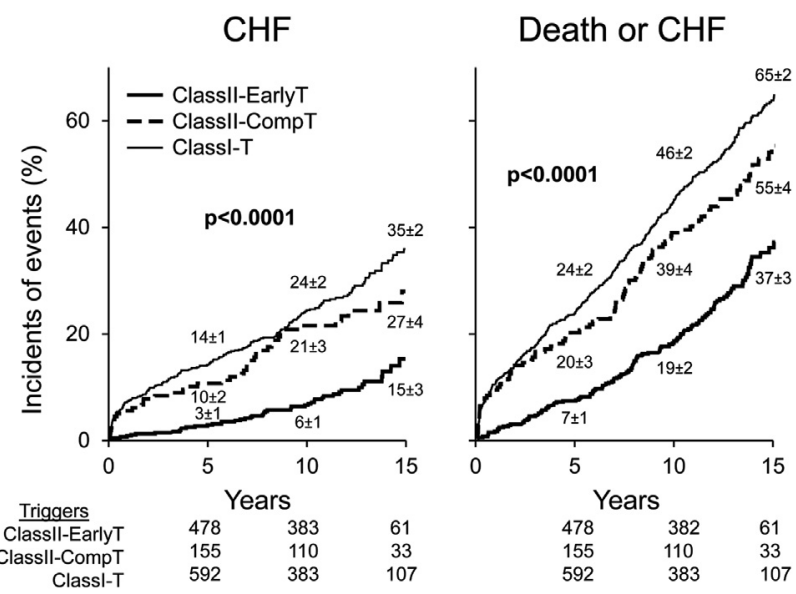

FIGURE 2. Incidence of postoperative CHF (left) or composite end point of heart failure or death (right) after surgery for severe organic MR, according to type of preoperative clinical triggers indicating mitral surgery. Triggers were classified as ClassI-T (heart failure symptoms, EF $<60 \%$, or end-systolic LV diameter $\geq 40 \mathrm{~mm}$ ), ClassII-CompT (atrial fibrillation or pulmonary hypertension), and ClassII-EarlyT on the basis of severe MR with high probability of valve repair in the absence of other class I or II triggers. The numbers at the bottom indicate the patients remaining at risk for each group and interval. The numbers along the curves indicate survival \pm standard error. Note the higher incidence of heart failure and of heart failure or death with ClassI-T (thin solid curve) and ClassII-CompT (thick dashed curve) compared with ClassII-EarlyT (early surgery; thick solid curve). $\mathrm{CHF}$, Congestive heart failure.

triggers at surgery in a large cohort of patients with severe organic MR. Our main result is that the type of guidelinebased trigger used to indicate surgery for organic MR is associated with major implications for short- and longterm outcomes after surgery. This result is observed in a framework of an "apples to apples" comparison obtained by accounting for identified confounders, particularly age, using multiple methods (standard Cox adjustment, age stratification, inverse probability weighting, comparison with expected survival) demonstrating that the impact of guideline-based indications on postoperative outcome is unadulterated and independent. The context of our study also makes these results particularly important. Indeed, this outcome penalty is noted in an era of high valve repair performance and low-risk surgery, making our conclusions applicable to current optimal practice. Notwithstanding the rarity of operative complications, ClassI-T at surgery implies more complications, particularly operative mortality. Long term after surgery, ClassI-T (heart failure symptoms, $\mathrm{EF}<60 \%$, or end-systolic diameter $\geq 40 \mathrm{~mm}$ ) indicates approximately double the risk of postoperative death/CHF versus early surgery (ClassII-EarlyT). Even ClassIICompT (atrial fibrillation or pulmonary hypertension), although less frequent as isolated indication, implies an excess risk of long-term death/CHF compared with early surgery (ClassII-EarlyT). Excess trigger-related risk persists even when analysis is restricted to valve repair or to older or younger patients. Although rescue surgery is warranted when patients first present with ClassI-T or ClassII-CompT, such surgical indications are associated with long-term decreased survival and increased heart failure. Thus, it is not desirable to defer surgery by choice to reach such triggers in severe organic MR unless operative risk and repair probability are poor. Conversely, early surgery in this era of low operative risk and high repair rates provides the best long-term outcomes after organic MR surgery. Distinguishing mandatory rescue surgery in the face of advanced presentation from preferred management choice in patients diagnosed early in the course of their valve disease should be included in clinical guidelines.

\section{Controversy Regarding Timing of Surgery for Organic Mitral Regurgitation}

Timing of surgery in organic MR is uncertain and controversial, ${ }^{3,4}$ focusing on divergent studies of the natural history of organic severe MR. ${ }^{19-22}$ Early data on degenerative MR demonstrating excess mortality under medical management ${ }^{19}$ have been countered by a small series of patients who, under so-called watchful waiting, demonstrated low mortality. ${ }^{22}$ This study was used to show that following patients up to the occurrence of ClassI-T or ClassII-CompT guideline-based triggers for surgery is safe and should be preferred. ${ }^{4}$ Several recent studies have contradicted this. The concept of safe follow-up within Europeantype health systems was negated by European collaborative studies showing high complication rates. ${ }^{20}$ Studies with MR quantitation showed that severe quantified MR yields excess mortality and morbidity under medical management. ${ }^{21}$ Outcome analysis according to age showed excess mortality specifically in older patients, ${ }^{23}$ reconciling the fact that small series of mostly young patients cannot demonstrate this mortality risk. Conversely, young patients incur a marked excess heart failure/atrial fibrillation risk and are also not safe. ${ }^{23}$ Although evidence tends toward high mortality and morbidity during organic MR medical follow-up and toward improved outcome with early surgery, ${ }^{24-27}$ the controversy is not over. The discord regards whether classic triggers for MR surgery-heart failure symptoms, atrial fibrillation, asymptomatic pulmonary hypertension, or LV dysfunction-indicate persistent excess postoperative risk or not. If surgical improvements, low operative mortality, and high repair rates minimize outcome consequences of surgical indications, the controversy would be resolved. Thus, it is essential to verify whether guideline-based triggers affect postoperative outcome in this era of improved MR surgery.

\section{Implications of Guideline-Based Surgical Triggers for Outcome}

Outcome after surgery for organic MR is determined by repair, ${ }^{28}$ coronary disease, ${ }^{29}$ and comorbidity, but early 
studies suggested that patients' preoperative status may contribute importantly to mortality and morbidity after surgical correction of MR. ${ }^{6}$ Preoperative characteristics, mentioned by guidelines in Europe and the United States as surgical triggers, demonstrate little difference. ${ }^{1,2}$ End-systolic LV diameter thresholds of $40 \mathrm{~mm}$ in US guidelines and 40 to $45 \mathrm{~mm}$ in European guidelines depending on MR cause ${ }^{2}$ and classification of early surgery as class IIa (United States) versus class IIb (Europe) represent minor discrepancies. ${ }^{1,2}$ Minor variations on new indices are not yet generalized, ${ }^{30}$ and the backbone of class I and II surgical triggers is common, as in our study criteria.

Early work suggested that symptoms before surgery implied reduced survival postoperatively. ${ }^{6,8,14}$ Likewise, decreased EF was associated with excess postoperative mortality. ${ }^{5,9}$ More recently, there have been suggestions that risk associated with an end-systolic diameter of $40 \mathrm{~mm}$ or more ${ }^{10}$ and pulmonary hypertension ${ }^{31}$ may persist postoperatively. However, outcome implications of guideline-based triggers were not examined comprehensively, and most information came from an era of frequent valve replacement. Valve repair is associated with improved survival and LV function, ${ }^{28}$ and repair rates markedly increased with profound declines in operative mortality. ${ }^{13}$ Previously noted consequences of individual surgical triggers may have been eradicated in the valve repair era. Thus, our study period from 1990 to 2000 provided both the valve repair era and long-term follow-up.

We observe that, despite surgical improvements and in all subsets of patients, ${ }^{32}$ ClassI-T and ClassII-CompT profoundly and independently affect short- and long-term outcomes. Early postoperative excess complications are relatively modest, notable with ClassI-T, and should not lead to denial of rescue surgery. ${ }^{16,33}$ Long-term excess risk affirmed by multiple methods balancing baseline characteristics was notable with ClassII-CompT and considerable with ClassI-T. Mechanistically, excess complications with lower EF and higher LV end-systolic diameter are linked to postoperative LV dysfunction, ${ }^{34,35}$ a known cause of excess mortality. ${ }^{5,36}$ Preoperative symptoms improve postoperatively but reflect subtle alterations of cardiac compliance ${ }^{37,38}$ and neurohormonal imbalance ${ }^{39}$ that may persist postoperatively with excess postoperative mortality. ${ }^{6,14}$ Thus, despite widespread valve repair and low operative risk, aggregate preoperative ClassI-T implies considerable excess postoperative risk. ClassII-CompT, atrial fibrillation, ${ }^{11}$ and pulmonary hypertension, ${ }^{31}$ although considered less severe, are linked by our study to excess long-term death/CHF and are not benign indications. $^{12}$ Thus, reaching guideline-based triggers, ClassI-T or ClassII-CompT is not safe, imposes postoperative excess complications, and has important implications.

\section{Implications for Clinical Practice}

Excess postoperative risk with severe MR and ClassI-T or ClassII-CompT should not lead to the conclusion that patients do not benefit from surgical correction. Indeed, patients benefit from relief of symptoms and cardiac overload at low operative risk with a high probability of valve repair. ${ }^{33,40}$ Of note, left unoperated, such patients incur considerable mortality, including sudden death. ${ }^{19,41}$ Thus, rescue surgery is unquestionably warranted, providing 5-year survival free of CHF in more than $75 \%$ of cases (vs $<25 \%$ under medical management ${ }^{19}$ ). However, wide agreement that rescue surgery is necessary does not imply that ClassI-T is the preferred timing for MR surgery. Surgical outcome with ClassII-CompT also is an improvement versus that with medical treatment, ${ }^{11,42}$ but these "less severe" triggers still imply rescue surgery with a suboptimal outcome.

Conversely, surgery in those with ClassII-EarlyT (ie, early surgery for severe MR) performed before clinical triggers develop ${ }^{32}$ provides the best operative outcome and lowest mortality/CHF. ${ }^{43}$ This approach is advisable because perioperative complications are infrequent and repair is primarily the rule ${ }^{32}$ even in this diverse population, including those with rheumatic or endocarditic valves. However, nationwide, MR surgery is heterogeneous, with low-volume centers affected by higher operative mortality and lower repair rates, ${ }^{18,44}$ so the early surgical approach should be reserved for advanced valve repair centers that take advantage of high volume to ensure excellent imaging, frequent repair of proven durability, and low operative mortality. High-quality services are essential to apply the early surgery component of guidelines. ${ }^{1}$ Multiple objective markers ${ }^{21,30,37,39}$ of outcome under medical management allow the detection of patients in whom surgical risk, even minimal, is acceptable.

\section{Study Limitations}

Affirming differences in outcome is theoretically best addressed by clinical trials with baseline characteristics identical between strategies. Although age in our series is that generally noted at surgery, ${ }^{18}$ patients who underwent operation with ClassI-T or ClassII-CompT were older with a longer duration of murmur in our series, representing a potential bias. However, no clinical trial can randomly assign clinical characteristics at presentation of patients before surgery, so comparing outcomes according to clinical preoperative presentation cannot be achieved by a clinical trial. Multiple methods of accounting for differences in baseline characteristics, including standard Cox method adjustment, stratification, IPW, and adjustment for expected survival, all concur in demonstrating an independent impact on the postoperative outcome of these triggers. Obviously, what defines the triggers cannot be 
adjusted for because of tautological implications, but major predictors of outcome were accounted for, demonstrating that ClassI-T and ClassII-CompT indicate suboptimal postoperative outcomes. Registries such as the STS provide large samples to assess valve repair, ${ }^{45}$ but without MR cause or triggers for surgery, they cannot answer the question of outcome implications of surgical triggers in organic MR.

\section{CONCLUSIONS}

In the current era, despite high repair rates and low operative mortality, guideline-based triggers for MR surgery carry major independent risk implications for short- and long-term outcomes. ClassI triggers should be avoided because they imply higher operative mortality, postoperative long-term mortality (doubled vs ClassII-EarlyT), and heart failure rates. ClassII-CompT triggers are also serious, with higher long-term postoperative mortality and heart failure rates. Thus, both of these trigger types promptly necessitate rescue surgery to relieve patients of symptoms and volume overload, but nonetheless imply excess risk that continues to accrue long after MR surgery. In contrast, surgery in those with ClassII-EarlyT that is performed at valve centers providing low operative risk and high repair rates allows outstanding outcomes.

\section{Conflict of Interest Statement}

Authors have nothing to disclose with regard to commercial support.

\section{References}

1. Nishimura RA, Otto CM, Bonow RO, Carabello BA, Erwin JP III, Guyton RA, et al. 2014 AHA/ACC guideline for the management of patients with valvular heart disease: a report of the American College of Cardiology/American Heart Association task force on practice guidelines. Circulation. 2014;129:e521-643.

2. Vahanian A, Alfieri O, Andreotti F, Antunes MJ, Baron-Esquivias G, Baumgartner $\mathrm{H}$, et al. Guidelines on the management of valvular heart disease (version 2012). Eur Heart J. 2012;33:2451-96.

3. Enriquez-Sarano M, Sundt TM III. Early surgery is recommended for mitral regurgitation. Circulation. 2010;121:804-12.

4. Gillam LD, Schwartz A. Primum non nocere: the case for watchful waiting in asymptomatic "severe" degenerative mitral regurgitation. Circulation. 2010; 121:813-21.

5. Enriquez-Sarano M, Tajik A, Schaff H, Orszulak T, Bailey K, Frye R. Echocardiographic prediction of survival after surgical correction of organic mitral regurgitation. Circulation. 1994;90:830-7.

6. Tribouilloy C, Enriquez-Sarano M, Schaff H, Orszulak T, Bailey K, Tajik A, et al. Impact of preoperative symptoms on survival after surgical correction of organic mitral regurgitation: rationale for optimizing surgical indications. Circulation. 1999;99:400-5

7. Wisenbaugh T, Skudicky D, Sareli P. Prediction of outcome after valve replacement for rheumatic mitral regurgitation in the era of chordal preservation. Circulation. 1994;89:191-7.

8. David TE, Ivanov J, Armstrong S, Rakowski H. Late outcomes of mitral valve repair for floppy valves: implications for asymptomatic patients. J Thorac Cardiovasc Surg. 2003;125:1143-52.

9. Lee EM, Shapiro LM, Wells FC. Mortality and morbidity after mitral valve repair: the importance of left ventricular dysfunction. J Heart Valve Dis. 1995; 4:460-70.

10. Tribouilloy C, Grigioni F, Avierinos JF, Barbieri A, Rusinaru D, Szymanski C, et al. Survival implication of left ventricular end-systolic diameter in mitral regurgitation due to flail leaflets: a long-term follow-up multicenter study. $J$ Am Coll Cardiol. 2009;54:1961-8.

11. Eguchi K, Ohtaki E, Matsumura T, Tanaka K, Tohbaru T, Iguchi N, et al. Pre-operative atrial fibrillation as the key determinant of outcome of mitral valve repair for degenerative mitral regurgitation. Eur Heart J. 2005;26:1866-72.

12. Chenot F, Montant P, Vancraeynest D, Pasquet A, Gerber B, Noirhomme PH, et al. Long-term clinical outcome of mitral valve repair in asymptomatic severe mitral regurgitation. Eur J Cardiothorac Surg. 2009;36:539-45.

13. Detaint D, Sundt TM, Nkomo VT, Scott CG, Tajik AJ, Schaff HV, et al. Surgical correction of mitral regurgitation in the elderly: outcomes and recent improvements. Circulation. 2006;114:265-72.

14. Gillinov AM, Mihaljevic T, Blackstone EH, George K, Svensson LG, Nowicki ER, et al. Should patients with severe degenerative mitral regurgitation delay surgery until symptoms develop? Ann Thorac Surg. 2010;90:481-8.

15. O'Gara P, Sugeng L, Lang R, Sarano M, Hung J, Raman S, et al. The role of imaging in chronic degenerative mitral regurgitation. JACC Cardiovasc Imaging. 2008;1:221-37.

16. Chatterjee S, Rankin JS, Gammie JS, Sheng S, O’Brien SM, Brennan JM, et al. Isolated mitral valve surgery risk in 77,836 patients from the Society of Thoracic Surgeons database. Ann Thorac Surg. 2013;96:1587-95.

17. Ho K, Anderson K, Kannel W, Grossman W, Levy D. Survival after the onset of congestive heart failure in Framingham Heart Study subjects. Circulation. 1993; $88: 107-15$.

18. Gammie JS, O'Brien SM, Griffith BP, Ferguson TB, Peterson ED. Influence of hospital procedural volume on care process and mortality for patients undergoing elective surgery for mitral regurgitation. Circulation. 2007;115:881-7.

19. Ling H, Enriquez-Sarano M, Seward J, Tajik A, Schaff H, Bailey K, et al. Clinical outcome of mitral regurgitation due to flail leaflets. N Engl J Med. 1996;335: $1417-23$.

20. Grigioni F, Tribouilloy C, Avierinos JF, Barbieri A, Ferlito M, Trojette F, et al Outcomes in mitral regurgitation due to flail leaflets. J Am Coll Cardiol Img. 2008;1:133-41.

21. Enriquez-Sarano M, Avierinos JF, Messika-Zeitoun D, Detaint D, Capps M, Nkomo V, et al. Quantitative determinants of the outcome of asymptomatic mitral regurgitation. N Engl J Med. 2005;352:875-83.

22. Rosenhek R, Rader F, Klaar U, Gabriel H, Krejc M, Kalbeck D, et al. Outcome of watchful waiting in asymptomatic severe mitral regurgitation. Circulation. 2006 113:2238-44.

23. Avierinos JF, Tribouilloy C, Grigioni F, Suri R, Barbieri A, Michelena HI, et al Impact of ageing on presentation and outcome of mitral regurgitation due to flail leaflet: a multicentre international study. Eur Heart J. 2013;34:2600-9.

24. Suri RM, Grewal J, Mankad S, Enriquez-Sarano M, Miller FA Jr, Schaff HV. Is the anterior intertrigonal distance increased in patients with mitral regurgitation due to leaflet prolapse? Ann Thorac Surg. 2009;88:1202-8.

25. Kang DH, Kim JH, Rim JH, Kim MJ, Yun SC, Song JM, et al. Comparison of early surgery versus conventional treatment in asymptomatic severe mitral regurgitation. Circulation. 2009;119:797-804.

26. Ling L, Enriquez-Sarano M, Seward J, Orszulack T, Schaff H, Bailey K, et al. Early surgery in patients with mitral regurgitation due to partial flail leaflet: a long-term outcome study. Circulation. 1997;96:1819-25.

27. Montant P, Chenot F, Robert A, Vancraeynest D, Pasquet A, Gerber B, et al Long-term survival in asymptomatic patients with severe degenerative mitra regurgitation: a propensity score-based comparison between an early surgica strategy and a conservative treatment approach. J Thorac Cardiovasc Surg. 2009; 138:1339-48

28. Enriquez-Sarano M, Schaff HV, Orszulak TA, Tajik AJ, Bailey KR, Frye RL. Valve repair improves the outcome of surgery for mitral regurgitation. Circulation. 1995;91:1022-8.

29. Tribouilloy C, Enriquez-Sarano M, Schaff H, Orszulak T, Fett S, Bailey K, et al Excess mortality due to coronary artery disease after valvular surgery: secular trends in valvular regurgitation and effect of internal mammary bypass. Circulation. 1998;98:II108-15.

30. Le Tourneau T, Messika-Zeitoun D, Russo A, Detaint D, Topilsky Y, Mahoney DW, et al. Impact of left atrial volume on clinical outcome in organic mitral regurgitation. J Am Coll Cardiol. 2010;56:570-8.

31. Barbieri A, Bursi F, Grigioni F, Tribouilloy C, Avierinos JF, Michelena HI, et al Prognostic and therapeutic implications of pulmonary hypertension complicating degenerative mitral regurgitation due to flail leaflet: a multicenter long-term international study. Eur Heart J. 2010;32:751-9.

32. Suri RM, Vanoverschelde JL, Grigioni F, Schaff HV, Tribouilloy C, Avierinos JF, et al. Association between early surgical intervention vs watchful waiting and 
outcomes for mitral regurgitation due to flail mitral valve leaflets. JAMA. 2013; 310:609-16.

33. Atluri P, Woo YJ, Goldstone AB, Fox J, Acker MA, Szeto WY, et al. Minimally invasive mitral valve surgery can be performed with optimal outcomes in the presence of left ventricular dysfunction. Ann Thorac Surg. 2013;96:1596-602.

34. Enriquez-Sarano M, Tajik A, Schaff H, Orszulak T, McGoon M, Bailey K, et al Echocardiographic prediction of left ventricular function after correction of mitral regurgitation: Results and clinical implications. J Am Coll Cardiol. 1994;24:1536-43.

35. Suri RM, Schaff HV, Dearani JA, Sundt TM III, Daly RC, Mullany CJ, et al. Determinants of early decline in ejection fraction after surgical correction of mitral regurgitation. J Thorac Cardiovasc Surg. 2008;136:442-7.

36. Crawford M, Souchek J, Oprian C, Miller D, Rahimtoola S, Giacomini J, et al. Determinants of survival and left ventricular performance after mitral valve replacement. Circulation. 1990;81:1173-81.

37. Messika-Zeitoun D, Johnson BD, Nkomo V, Avierinos JF, Allison TG, Scott C, et al. Cardiopulmonary exercise testing determination of functional capacity in mitral regurgitation: Physiologic and outcome implications. J Am Coll Cardiol. 2006; 47:2521-7.

38. Kraus F, Dacian S, Hall D, Klein U, Rudolph W. Relationship between symptoms and hemodynamics associated with regurgitant lesions of the aortic or mitral valve. Z Kardiol. 1986;75:137-40

39. Pizarro R, Bazzino OO, Oberti PF, Falconi M, Achilli F, Arias A, et al. Prospective validation of the prognostic usefulness of brain natriuretic peptide in asymptomatic patients with chronic severe mitral regurgitation. J Am Coll Cardiol. 2009;54:1099-106.
40. Castillo JG, Anyanwu AC, Fuster V, Adams DH. A near 100\% repair rate for mitral valve prolapse is achievable in a reference center: implications for future guidelines. J Thorac Cardiovasc Surg. 2012;144:308-12.

41. Grigioni F, Enriquez-Sarano M, Ling L, Bailey K, Seward J, Tajik A, et al. Sudden death in mitral regurgitation due to flail leaflet. J Am Coll Cardiol. 1999:34:2078-85.

42. Grigioni F, Avierinos JF, Ling LH, Scott CG, Bailey KR, Tajik AJ, et al. Atrial fibrillation complicating the course of degenerative mitral regurgitation. Determinants and long-term outcome. J Am Coll Cardiol. 2002; 40:84-92.

43. Coutinho GF, Garcia AL, Correia PM, Branco C, Antunes MJ. Long-term follow-up of asymptomatic or mildly symptomatic patients with severe degenerative mitral regurgitation and preserved left ventricular function. J Thorac Cardiovasc Surg. 2014;148:2795-801.

44. Vassileva CM, McNeely C, Spertus J, Markwell S, Hazelrigg S. Hospital volume, mitral repair rates, and mortality in mitral valve surgery in the elderly: an analysis of us hospitals treating Medicare fee-for-service patients. $J$ Thorac Cardiovasc Surg. 2015;149:762-8.e1.

45. Badhwar V, Peterson ED, Jacobs JP, He X, Brennan JM, O'Brien SM, et al. Longitudinal outcome of isolated mitral repair in older patients: results from 14,604 procedures performed from 1991 to 2007. Ann Thorac Surg. 2012;94: 1870-9.

Key Words: guidelines, heart failure, mitral regurgitation, surgery, survival, valve repair

Readers who found these articles interesting may also like to read the following papers found in recent and future issues of our sister publications, Seminars in Thoracic and Cardiovascular Surgery and Operative Techniques in Thoracic and Cardiovascular Surgery!

\section{Acquired Cardiovascular Disease: Valvular Heart Disease}

Current Readings: Tirone David. Aortic Valve Sparing Operations. Semin Thorac Cardiovasc Surg. Autumn 2014;26(3):231-238.

News and Views: Rakesh Suri. Mitral Valve Repair in Asymptomatic Patients with Severe Mitral Regurgitation: Pushing Past the Tipping Point. Semin Thorac Cardiovasc Surg. Summer 2014;26(2):95-101.

News and Views: Michael Reardon. The CoreValve US Pivotal Trial. Semin Thorac Cardiovasc Surg. Autumn 2014;26(3):179-86.

Ravi Ghanta. Staged Hybrid Repair for Extent II Thoracoabdominal Aortic Aneurysms and Dissections. Oper Tech Thorac Cardiovasc Surg. Summer 2014;19(2):238-251.

Gorav Ailawadi. Transcatheter MV repair. Oper Tech Thorac Cardiovasc Surg. Summer 2014;19(2):219-237. 